\title{
Plasma concentrations of dasatinib have a clinical impact on the frequency of dasatinib dose reduction and interruption in chronic myeloid leukemia: an analysis of the DARIA 01 study
}

\author{
Shuichi Mizuta ${ }^{1,11}$ (D) Masashi Sawa ${ }^{2} \cdot$ Hisashi Tsurumi ${ }^{3} \cdot$ Kana Matsumoto $^{4} \cdot$ Kotaro Miyao $^{2} \cdot$ Takeshi Hara $^{3} \cdot$ \\ Takeshi Takahashi $^{5}$ - Reona Sakemura ${ }^{2} \cdot$ Hiroshi Kojima $^{6}$ - Akio Kohno ${ }^{7}$ Mari S. Oba ${ }^{8}$ Satoshi Morita ${ }^{9}$. \\ Junichi Sakamoto ${ }^{10} \cdot$ Nobuhiko Emi $^{1}$
}

Received: 16 March 2018 / Accepted: 24 May 2018 / Published online: 29 May 2018

(c) The Author(s) 2018

\begin{abstract}
Background Dasatinib has shown promising anti-leukemic activity against chronic myeloid leukemia (CML). However, patients receiving dasatinib frequently require dose reductions and treatment interruptions (treatment alteration).

Methods We prospectively analyzed the frequency and significance of treatment alteration during dasatinib therapy in patients with CML. In all patients, trough plasma concentrations of dasatinib $\left(C_{\min }\right)$ at steady state were assessed on day 28 of therapy.

Results $28 \%$ of patients had their doses reduced at a median of 42 days, and $25 \%$ of patients had temporarily interrupted at a median of 54 days after treatment initiation. The overall dasatinib treatment alteration-free rate at 1 year was $66 \%$. Age was significantly correlated with $C_{\min }$ on day $28(p=0.014)$, and the correlation remained significant after adjusting dasatinib dose $(\mathrm{g})$, body weight $(\mathrm{kg})\left(C_{\mathrm{min}} / D / W\right)(p=0.026)$. In the univariate analysis, deep molecular response, advanced PS, higher $C_{\min } / D / W$ were associated with a significantly higher risk of treatment alteration (HR 4.19, 95\% CI: $1.06-16.60, p=0.041$; HR 5.26, 95\% CI: 1.33-20.80, $p=0.018$; and HR 10.15, 95\% CI: 2.55-40.48, $p=0.001$, respectively). In the multivariate analysis, advanced PS and higher $C_{\min } / D / W$ were correlated with the incidence of treatment alteration (HR $4.78,95 \%$ CI: 1.01-22.70, $p=0.049$; HR 6.17, 95\% CI: 1.17-32.50, respectively).

Conclusion Current data demonstrate that patients treated with dasatinib who displayed a high $C_{\mathrm{min}} / D / W$ value and/or advanced PS were at a high risk for altered treatment.
\end{abstract}

Keywords Dasatinib · Plasma concentration $\cdot$ Chronic myeloid leukemia $\cdot$ Treatment adherence $\cdot$ Individualized dasatinib therapy

\section{Introduction}

Dasatinib is a novel tyrosine kinase inhibitor (TKI) of $B C R$ $A B L$ and $S R C$ family kinases that has shown promising therapeutic effects in patients with chronic myeloid leukemia (CML) [1-6]. In the DASISION study, the cumulative rates of major molecular response (MMR) and molecular response at 5 years were significantly greater in patients treated with dasatinib compared with imatinib [4-6]. However, there were no significant differences in progression-free survival

Shuichi Mizuta

mizuta@spacelan.ne.jp

Extended author information available on the last page of the article and overall survival (OS) at 5 years [6]. In the DASISION study, $39 \%$ of patients initially treated with dasatinib and $37 \%$ of patients treated with imatinib are no longer receiving their respective initial therapy, and this discontinuation might have limited the potential benefits of treatment [6]. With regard to the imatinib therapy, poor adherence seemed to be the predominant reason underlying the lack of adequate clinical responses [7]. Larson et al. focused on the imatinib pharmacokinetics and reported that plasma trough levels of imatinib at steady state (day 29) was a significant prognostic indicator of midterm and long-term clinical responses in CML patients [8]. Then, we prospectively analyzed the frequency and significance of dose reductions and treatment interruptions during dasatinib therapy (treatment alteration) 
in patients with CML focusing on trough plasma concentrations of dasatinib at steady state.

\section{Materials and methods}

\section{Patients}

We conducted a phase 2 study to evaluate continuity of dasatinib therapy in patients with chronic phase CML (CML-CP) in the DARIA 01 study (UMIN000007345). Patients enrolled in the study were at least 16 years of age, and had Ph-positive CML-CP and primary or acquired hematologic resistance or intolerance to prior TKI therapy (imatinib and/or nilotinib). CML-CP was defined as $<15 \%$ blasts, $<20 \%$ basophils, $<30 \%$ blasts and promyelocytes, and platelets $>100 \times 10^{9} / \mathrm{L}$ in peripheral blood samples, and no extramedullary involvement. Patients were considered TKI-intolerant if they had previously only tolerated TKI doses less than $400 \mathrm{mg} /$ day or had discontinued TKI therapy due to toxicity potentially related to imatinib at a dose of $400 \mathrm{mg} /$ day or less. Primary resistance to prior TKI therapy was defined as lack of complete hematologic response (CHR) after 3 months, lack of major cytogenetic response (MCyR) after 6 months, and lack of complete cytogenetic response (CCyR) after 12 months. The present study was approved by the Institutional Review Board of the Fujita Health University School of Medicine and conformed to the provisions of the 1964 Declaration of Helsinki and its later amendments or comparable ethical standards. All patients provided written, informed consent before participating in the study.

\section{Study design and treatment}

The primary objective of this study was to identify factors influencing the frequency and significance of treatment alteration during dasatinib therapy at 12 months. The secondary objective was to evaluate the correlation between pharmacokinetic (PK) parameters and treatment alteration of dasatinib therapy, adverse events (AEs). Dasatinib was administered at an initial dose of $100 \mathrm{mg}$ once daily. Dose interruption or reduction was permitted in cases of nonhematologic toxicity grade 2 or greater, hematologic toxicity grade 3 or greater, or upon request from a patient. After a dose interruption or reduction associated with toxicity, dasatinib could be re-administrated at a dose of 50-100 mg/ day if the toxicity recovered to Grade 1 or 0 . Dasatinib dose adjustment upon re-administration was determined by the treating physician. Treatment was continued until disease progression or intolerable toxicity. During the study, CML therapies other than dasatinib were prohibited; however, patients were permitted to receive hydroxyurea to control elevated WBC and/or platelets.

\section{Evaluations}

Patients were seen once-weekly for the first 4 weeks and once-monthly for the following 11 months. Treatment efficacy was determined on the basis of hematologic assessments, bone marrow cytogenetics, and molecular responses in peripheral blood as assessed every 3 months. Patients who achieved CCyR were not required to undergo bone marrow cytogenetic analysis. AEs were continuously assessed throughout the study, and they were graded according to National Cancer Institute Common Terminology Criteria for Adverse Events (CTCAE), version 3.0. Safety assessments included AEs, hematologic and cardiac enzyme levels, biochemical parameters, urinalysis, electrocardiography, and physical examination. Chest X-rays were conducted at baseline and once-weekly for the first 4 weeks. Following the first 4 weeks of the study, chest X-rays were conducted as required for the detection or monitoring of pleural effusion. All patients underwent $B C R-A B L$ mutational analysis at baseline. mRNA from peripheral blood cells was collected and analyzed for $B C R-A B L$ gene mutations using denaturing high-performance liquid chromatography and sequencing. $B C R-A B L$ transcripts were analyzed by Biomedical Laboratories (BML) (Tokyo, Japan) according to the international scale (IS) [9]. A deep molecular response (DMR) was defined as less than $0.01 \%$ of the International Scale (BCR-ABL IS), corresponding to a 4-log reduction from a standardized baseline value. Progression was defined as a doubling of white blood cell count, loss of CHR, increase in $\mathrm{Ph}$-positive bone marrow metaphase cells, transformation to accelerated phase/blast phase, or death from any cause. All patients were told that their status of dasatinib administration would be monitored, and they were required to declare unused tablets to their physician.

\section{PK analysis}

Trough plasma concentrations of dasatinib $\left(C_{\min }\right)$ at steady state were assessed on day 28 of therapy. Blood samples were collected in heparinized tubes and immediately centrifuged. The separated plasma samples were stored in polypropylene tubes at $-30{ }^{\circ} \mathrm{C}$ until the PK analysis. Plasma concentration of dasatinib was measured using high-performance liquid chromatography coupled with electrospray mass spectrometry (HPLC-MS) as previously described, with some modifications [10]. The lower limit of quantitation of dasatinib by this method was $0.1 \mathrm{ng} / \mathrm{mL}$ and intraand inter-day variabilities were within $5.0 \%$. 


\section{Data analysis and statistical methods}

Dasatinib dose reduction-free and/or interruption (treatment alteration) -free rates were estimated using the Kaplan-Meier product limit method. Differences between groups were analyzed using the log-rank test, the Fisher exact test was used to calculate $p$ values for incidences of responses and pleural effusion. Univariate and multivariate Cox regression analyses were used to evaluate the predictive value of various clinical variables on the risk of treatment alteration. The following variables were evaluated: age range $(<60$ or $\geq 60)$, CML status at dasatinib initiation (CP, CCyR, or DMR), CML profile (newly diagnosed, prior TKI intolerance, or prior TKI resistance), performance status (PS) at diagnosis (0 or 1$), C_{\min }$ on day $28(<1.4$ or $\geq 1.4 \mathrm{ng} / \mathrm{mL}), C_{\min }$ on day 28 after adjusting dasatinib dose $(\mathrm{g})$, body weight $(\mathrm{kg})\left(C_{\min } / D / W\right)$. The relationship between $C_{\min }, C_{\min } / D / W$, and age were evaluated using Pearson's correlation coefficient. All of the statistical analyses were conducted using STATA 12 software (STATA Corp., College Station, TX, USA).

\section{Results}

\section{Patients}

Between April 2012 and September 2013, 32 CML-CP patients were enrolled in the study. Patient characteristics are summarized in Table 1. The median age was 51 years (range 20-86). Twenty-three patients (75.0\%) were treatment-naïve, and the remaining 9 patients $(25.0 \%)$ had been switched to dasatinib from another TKI due to treatment intolerance or resistance. Dasatinib treatment was initiated a median of 1 month (range 0-109 months) after CML diagnosis. Upon the initiation of dasatinib, 26 patients were in the $\mathrm{CP}, 2$ were in CCyR, and 4 were in DMR. No patients had $B C R-A B L$ mutations at baseline.

\section{Toxic effects}

Grade 3 or 4 neutropenia and/or thrombocytopenia occurred in $9(28 \%)$ and 5 patients (16\%), respectively. Hematological AEs requiring dasatinib dose reduction and/or treatment interruption occurred in 5 patients (16\%), and these AEs typically resolved within 3 months. Grade 1 pleural effusion (PE) occurred in 3 patients (9\%), and grade 2 PE occurred in 5 patients (16\%). PE was treated with diuretics in 3 patients (38\%), steroid therapy and dasatinib dose reduction in 2 patients $(25 \%)$, diuretics and dasatinib dose reduction in 2 patients (25\%), and dasatinib dose reduction alone in 1
Table 1 Patient demographics and characteristics at baseline

\begin{tabular}{ll}
\hline Median age, years (range) & 51 (20-86) \\
Sex & $21 / 11$ \\
Male/female & \\
ECOG performance status & 29 \\
0 & 3 \\
1 & \\
Disease history & 23 \\
Newly diagnosed & 9 \\
Resistant/Intolerant & \\
Previous therapy for CML & 5 \\
Imatinib & 3 \\
Nilotinib & 1 \\
Other & 23 \\
None & \\
Disease status & 26 \\
CP & 2 \\
CCyR & 4 \\
DMR & \\
$B C R-A B L$ mutation status & $0 / 32$ \\
Positive/negative & \\
\hline$C P$ chronic phase; $C C y R$ cytogenetic complete remission; $D M R$ deep \\
molecular remission
\end{tabular}

patient (13\%). Complete clinical and radiological resolution of PE was achieved in 5 patients (62\%), and clinical symptoms resolved in the remaining 3 patients (38\%).

\section{Dasatinib interruption and dose reduction}

Figure 1 details the progress of all patients through the study. The dose of dasatinib was reduced in 9 patients (28\%) at a median of 42 days (range 7-123 days) after treatment initiation. Treatment was temporarily interrupted in 8 patients (25\%) at a median of 54 days (range 14-331 days) after treatment initiation. Among 8 patients who had PE, 5 patients experienced treatment alteration (reduction in 2 patients, reduction and interruption in 3 patients). Three patients (9\%) discontinued treatment due to withdrawal of consent at 287 day, myelo-suppression at 236 days, or gastro-intestinal bleeding at 342 days. At the final observation, 25 patients were being treated with $100 \mathrm{mg}$ of dasatinib, and 4 patients were being treated with a reduced dose $(50 \mathrm{or} 40 \mathrm{mg})$. The overall treatment alteration-free rate was $66 \%$ (95\% confidence interval [CI]: 47-79\%) (Fig. 2).

\section{Clinical response}

The rates of CCyR and DMR in the 22 treatment-naïve patients were $68 \%$ and $4 \%$ at 3 months, $91 \%$ and $32 \%$ at 6 months, and $91 \%$ and $55 \%$ at 12 months, respectively. In the 10 patients who had been switched from other TKIs, 3 of 
Fig. 1 A flowchart of all patients entered into the DARIA 01 study

\section{A flowchart of all patients entered into the DARIA01 study}

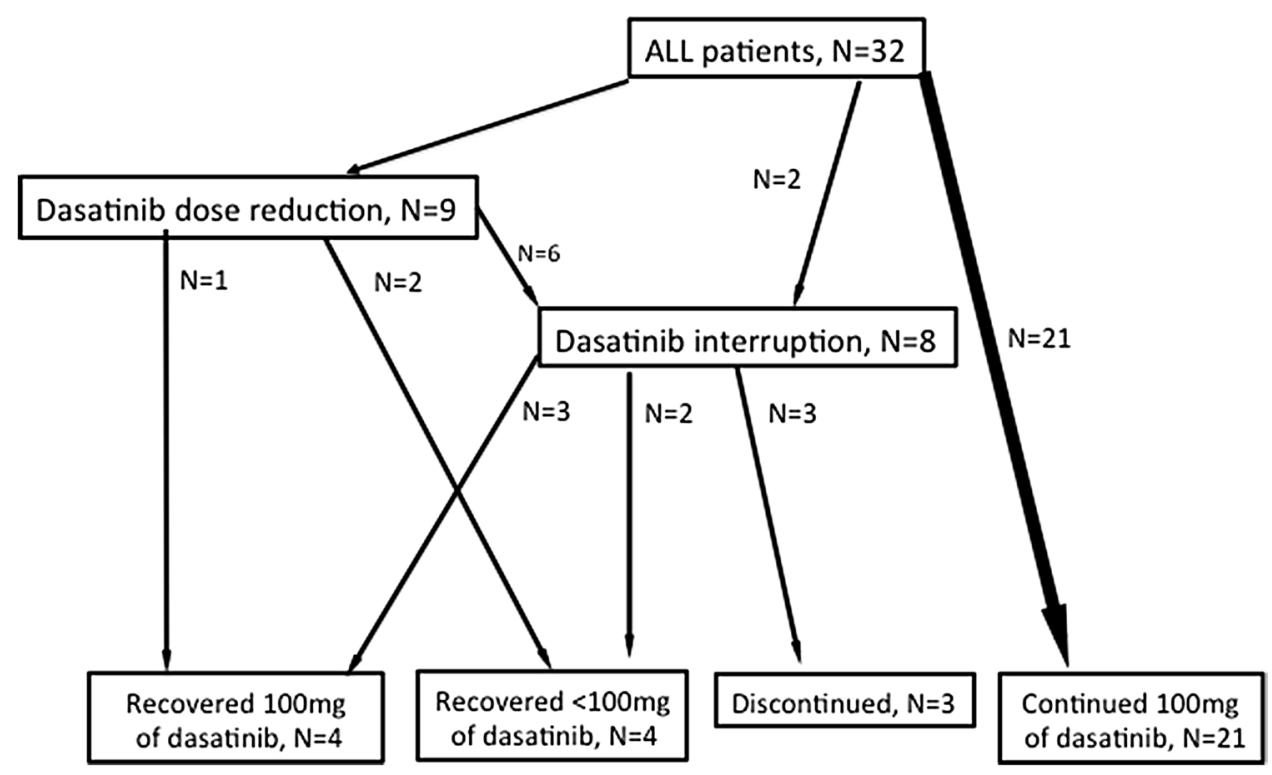

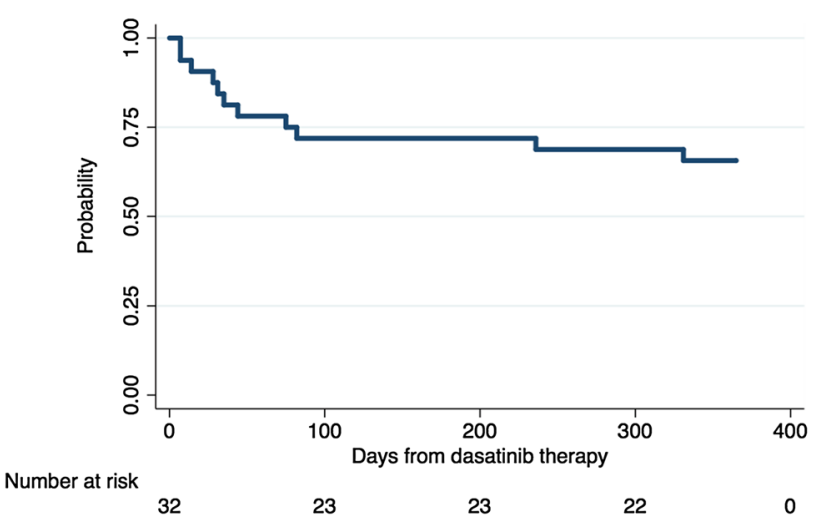

Fig. 2 Dasatinib dose reduction- and/or interruption-free (alterationfree) rate

the 4 patients in CHR achieved CCyR, and 1 of 2 patients in CCyR achieved a DMR at 3 months. The rates of CCyR and DMR were $90 \%$ and $60 \%$ at 3 months, $90 \%$ and $60 \%$ at 6 months, and $90 \%$ and $78 \%$ at 12 months, respectively. Twenty-nine of the 32 patients remained on dasatinib therapy at 1 year. No patients experienced disease progression while being treated with dasatinib. There was no significant difference in clinical responses in patients that underwent treatment alteration $(p=0.157)$ compared with patients that did not undergo these adjustments.

\section{Correlation of dasatinib trough levels with age}

PK data associated with dasatinib on day 28 were available for all of the study participants. On day 28, 27 patients were still being treated with the initial dose of $100 \mathrm{mg}$, and the remaining 5 were being treated with a lower dose $(50 \mathrm{mg})$ due to an AE. The median $C_{\min }$ of dasatinib was $1.4 \mathrm{ng} /$ $\mathrm{mL}$ (range $0.0-6.0 \mathrm{ng} / \mathrm{mL}$ ). The median $C_{\min }$ after adjusting for dasatinib dose $(\mathrm{g})$ and body weight $(\mathrm{kg})\left(C_{\min } / D / W\right)$, on day 28 was 0.19 (range 0.00-2.7). The inter-patient variability might reflect individual differences in drug metabolism and/or excretion. A linear regression analysis demonstrated that age was significantly correlated with $C_{\min }$ on day 28 $(r=0.431, p=0.014$; Fig. 3), and the correlation remained significant after adjusting for $C_{\min } / D / W(r=0.394, p=0.026$; Fig. 3).

\section{PE and dasatinib serum concentration}

Among 16 patients who showed high $C_{\min }(\geq 1.4 \mathrm{ng} / \mathrm{mL})$, five (31\%) experienced PE. On the other hand, in 16 patients who showed low $C_{\min }$ ( $<1.4 \mathrm{ng} / \mathrm{mL}$ ), 3 (19\%) experienced $\mathrm{PE}$. There was no significance in the incidence of $\mathrm{PE}$ between two groups $(p=0.685)$. Among 16 patients who showed high $C_{\min } / D / W(\geq 0.19)$, six (38\%) experienced PE. On the other hand, in 16 patients who showed low $C_{\min } / D / W$ $(<0.19), 2$ (13\%) experienced PE. The difference between two groups did not reach statistical difference $(p=0.220)$. The incidence of PE by 12 months in high age $(\geq 60)$ group was higher than that in low age $(<60)$ group, but did not 
Fig. 3 Relationship between age and dasatinib trough plasma concentrations

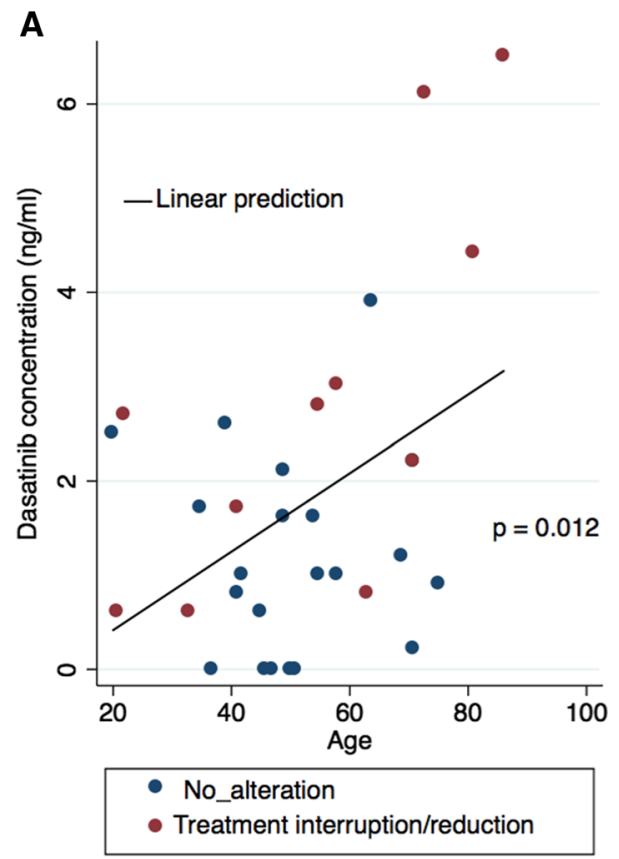

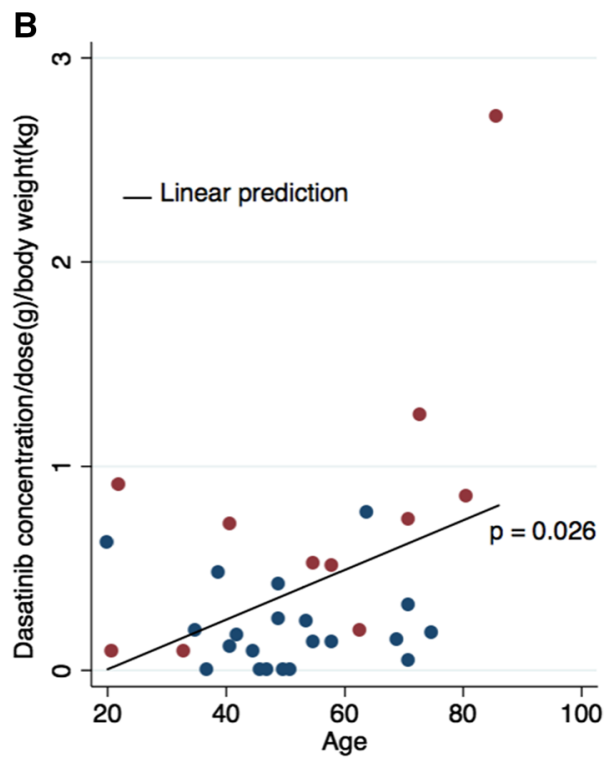

- No_alteration

- Treatment interruption/reduction reach statistical significance ( $40 \%$ vs. $18 \%, p=0.1864$ ). With regard to adverse events other than $\mathrm{PE}$, there were no significant correlation between AEs and dasatinib serum concentration. ( $C_{\min }, C_{\min } / D / W$ ) (Supplemental tables 1,2 ).

\section{Risk factors for dasatinib treatment alteration}

The treatment alteration-free rate was significantly greater in the low $C_{\min }$ group $(<1.4 \mathrm{ng} / \mathrm{mL})(81,95 \% \mathrm{CI}: 52-94 \%)$ compared with the high $C_{\min }$ group $(50,95 \% \mathrm{CI}: 25-71 \%$, $p=0.0473$ ) (Fig. 4). And also, the treatment alteration-free rate in low $C_{\min } / D / W$ group $(88,95 \% \mathrm{CI}: 59-97 \%)$ was significantly greater than that in the high $C_{\min } / D / W$ group (44, 95\% CI: 20-66\%, $p=0.0047$ ) (Fig. 4). Table 2 presents the results of the univariate and multivariate Cox regression analyses of risk factors for treatment alteration. In the univariate analysis, DMR, advanced PS, higher $C_{\min } / D / W$ were associated with a significantly higher risk of treatment alteration (HR 4.19, 95\% CI: 1.06-16.60, $p=0.041$; HR 5.26, 95\% CI: 1.33-20.80, $p=0.018$; and HR 10.15, 95\%
Fig. 4 a 1-year dasatinib treatment alteration-free rate by dasatinib trough plasma concentrations $\left(C_{\min }\right)(\mathrm{ng} /$ $\mathrm{mL})(1.4 \geqq$ vs. $<1.4)$. b 1 -year dasatinib treatment alterationfree rate by trough plasma concentrations of dasatinib after adjusting for dasatinib dose $(\mathrm{g})$ and body weight $(\mathrm{kg})\left(C_{\min } / D / W\right.$ ratio $)$ at day $28(0.19 \geqq$ vs. $<$ 0.19 )

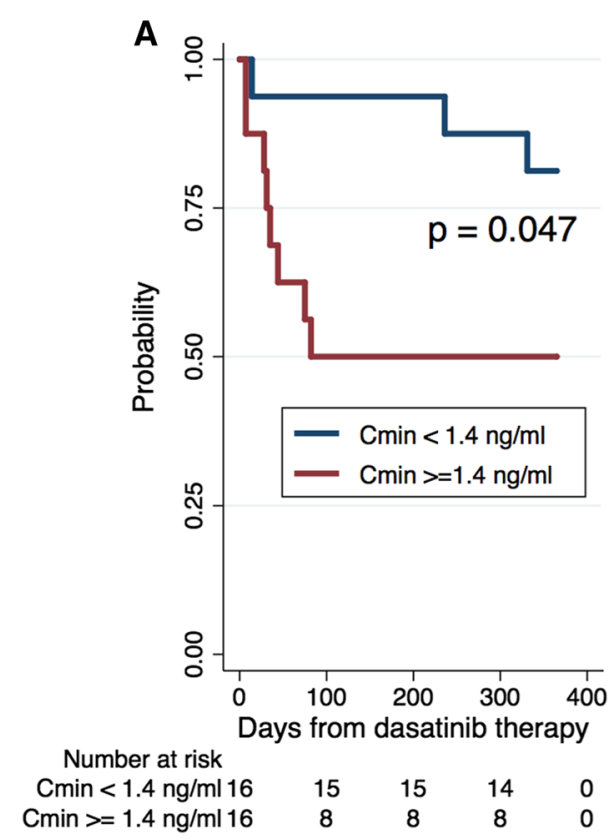


Table 2 Relationship between the dasatinib interruption/ reduction of treatment and the remaining variables

\begin{tabular}{|c|c|c|c|c|}
\hline \multirow[t]{2}{*}{ Characteristic } & \multicolumn{2}{|l|}{ Univariate analysis } & \multicolumn{2}{|c|}{ Multivariate analysis } \\
\hline & $\mathrm{HR}(95 \% \mathrm{CI})$ & $\mathrm{p}$ & $\operatorname{HR}(95 \% \mathrm{CI})$ & $p$ \\
\hline \multicolumn{5}{|l|}{ Age } \\
\hline$>60$ & 1.00 & & & \\
\hline$\geq 60$ & $2.46(0.75-8.14)$ & 0.138 & NA & \\
\hline \multicolumn{5}{|l|}{ Sex } \\
\hline Female & 1.00 & & & \\
\hline Male & $1.88(0.57-6.17)$ & 0.300 & NA & \\
\hline \multicolumn{5}{|c|}{ CML status at dasatinib initiation } \\
\hline $\mathrm{CP}$ & 1.00 & & & \\
\hline CyCR & $2.52(0.31-20.7)$ & 0.388 & $1.26(0.14-11.0)$ & 0.834 \\
\hline DMR & $4.19(1.06-16.6)$ & 0.041 & $1.21(0.26-5.74)$ & 0.809 \\
\hline \multicolumn{5}{|c|}{ Prior-therapy before dasatinib administration } \\
\hline No & 1.00 & & & \\
\hline Yes & $2.60(0.79-8.58)$ & 0.116 & NA & \\
\hline \multicolumn{5}{|c|}{ PS at dasatinib treatment } \\
\hline 0 & 1.00 & 0.018 & & \\
\hline 1 & $5.26(1.3-20.8)$ & & $4.78(1.01-22.7)$ & 0.049 \\
\hline \multicolumn{5}{|c|}{$C_{\min }$ on day $28(\mathrm{ng} / \mathrm{mL})$} \\
\hline$>1.4$ & 1.00 & & & \\
\hline$\geq 1.4$ & $3.53(0.93-13.41)$ & 0.063 & NA & \\
\hline \multicolumn{5}{|c|}{$C_{\min } / D / W$ on day 28} \\
\hline$>0.19$ & 1.00 & & & \\
\hline$\geq 0.19$ & $10.15(2.55-40.48)$ & 0.001 & $6.17(1.17-32.5)$ & 0.032 \\
\hline
\end{tabular}

$C P$ chronic phase; $C y C R$ cytogenetical complete remission; $D M R$ deep molecular remission; $C_{\text {min }}$ plasma concentrations of dasatinib at steady state; $C_{\min } / D / W, C_{\min }$ after an adjustment in the dasatinib dose and body weight
CI: $2.55-40.48, p=0.001$, respectively). In the multivariate analysis, advanced PS and higher $C_{\min } / D / W$ were still correlated with the incidence of treatment alteration (HR 4.78, 95\% CI: $1.01-22.70, p=0.049$; HR 6.17, 95\% CI: $1.17-32.50, p=0.032)$.

\section{Discussion}

It is now recognized that discontinuation of dasatinib therapy is a critical factor in the achievement and maintenance of an optimal response to dasatinib therapy [11]. In the DASISION study, dasatinib treatment was interrupted in $63 \%$ of patients, the dose of dasatinib was reduced in $31 \%$ of patients [5]. At the end of the 3-year observation period, $29 \%$ patients discontinued dasatinib therapy for various reasons (AE: 10\%, disease progression or treatment failure: $10 \%$, unrelated AE: $2 \%$, death: $2 \%$, or other reason: $5 \%$ ) [5]. However, the detailed course leading to discontinuation in each case has not been reported. To the best of our knowledge, there have been no previous reports regarding the timing of dasatinib reduction, interruption, and/or discontinuation in midterm observation. In the present study, $28 \%$ of patients experienced a dose reduction and $25 \%$ of patients experienced temporarily interruption of treatment after initiating dasatinib therapy. The 1 -year overall dasatinib treatment alteration-free rate was $66 \%$. $9 \%$ of patients discontinued the treatment due to withdrawal of consent or an $\mathrm{AE}$ at 1-year from dasatinib therapy. No patients experienced disease progression while being treated with dasatinib.

As it often necessitates treatment discontinuation, PE can limit the therapeutic efficacy of dasatinib [12-14]. On the other hand, several studies reported that the pleural effusion was common in elderly patients and did not affect any negative treatment results if that were clinically manageable [15, 16]. In the present study, the incidence of PE by 12 months in high age $(\geq 60)$ group was higher than that in low age $(<60)$ group, but did not reach statistical significance $(p=0.1864)$. Several studies have reported that a higher Cmax of dasatinib was associated with greater clinical response rates, and lower trough concentrations were associated with a lower risk of PE [12, 17-19]. In the prospective OPTIM dasatinib trial, patients with a dasatinib trough concentration of $-3 \mathrm{nM}(1.5 \mathrm{ng} / \mathrm{mL})$ at day 15 were randomized to the non-dose adjustment group or the dose adjustment group to obtain a $\mathrm{C} 0$ of $<3 \mathrm{nM}[20]$. The overall rates of pleural 
effusion at 36 months were $49 \%$ and $11 \%$, respectively, in the non-adjustment and adjustment groups, and the discontinuation rates of dasatinib therapy were $27 \%$ and $13 \%$, respectively $(p=0.008)$ [20]. They concluded that monitoring dasatinib PK parameters could help predict the risk of side effects and prevent discontinuation of dasatinib therapy [20]. In the present study, patients who had high $C_{\min } / D / W$ showed higher incidence of PE than that in low $C_{\min } / D / W$ group (38\% vs. $13 \%$, respectively), despite it did not reach statistical significance $(p=0.220)$. Indeed, among 8 patients who had PE, 5 patients experienced treatment alteration. However, pleural effusions were well managed effectively through administration of diuretics or steroid therapy and/or dasatinib dose modifications. After then, they could continue dasatinib therapy. Better management of PE might contribute to prevent discontinuation of dasatinib therapy. In the present study, age was significantly correlated with $C_{\text {min }}$ $(p=0.012)$ (Fig. 2), and the correlation remained significant after adjusting for $C_{\min } / D / W(p=0.026)$. The treatment alteration-free rate was significantly greater in the low $C_{\text {min }}$ group $(<1.4 \mathrm{ng} / \mathrm{mL})$ compared with the high $C_{\min }$ group ( $81 \%$ and $50 \%$, respectively, $p=0.0473$ ) (Fig. 4). And also, the treatment alteration-free rate in low $C_{\min } / D / W$ group was significantly greater than that in the high $C_{\min } / D / W$ group (88\% and 44\%, respectively, $p=0.0047$ ) (Fig. 4). The higher $C_{\min } / D / W$, instead of higher $C_{\min }$, was significantly correlated with the incidence dasatinib treatment alteration rate in the multivariate analysis. The $C_{\min } / D / W$ possibly could reflect patient's ability to metabolize and/or excretion dasatinib. A larger patient sample is needed to determine which would be better to predict the dasatinib treatment alteration and to prevent discontinuation of dasatinib therapy.

To our knowledge, this is the first prospective study evaluating prognostic factors that influence the treatment alteration of dasatinib therapy in patients with CML. However, the limitations of our study need to be considered. Our study was limited by the presence of residual confounding factors, both known and unknown.

The present study focused on clinical outcomes of 32 patients at the midterm point (1 year) of the observation period of the DARIA 01 study; however, dasatinib therapy can be continued for much longer periods of time. A longterm observation study including more large number of patients is needed to clarify the risks for the treatment alteration of dasatinib therapy.

\section{Conclusion}

We demonstrated that trough plasma concentrations of dasatinib after adjusting for dasatinib dose and body weight $\left(C_{\min } / D / W\right)$ at day 28 and performance status (PS) at diagnosis were risk factors for treatment alteration during the midterm point in the observation period of the DARIA 01 study. Monitoring dasatinib PK parameters could help to predict the risk of treatment alteration of dasatinib therapy and optimize the efficacy of dasatinib therapy.

Acknowledgements This study was supported by a non-profit organization Epidemiological and Clinical Research Information Network (ECRIN). The authors thank Yumi Miyashita at ECRIN for collecting the data.

Author contributions SM: planning study design and conducting study, writing of the manuscript, and final approval of the manuscript. MS: data collection, writing of the manuscript, and final approval of the manuscript. HT: data collection, writing of the manuscript, and final approval of the manuscript. KM: PK analysis, data interpretation and writing of the manuscript, and final approval of the manuscript. KM: data collection, writing of the manuscript, and final approval of the manuscript. TH: data collection, writing of the manuscript, and final approval of the manuscript. TT: data collection, writing of the manuscript, and final approval of the manuscript. RS: data collection, writing of the manuscript, and final approval of the manuscript. HK: data collection, writing of the manuscript, and final approval of the manuscript. AK: data collection, writing of the manuscript, and final approval of the manuscript. MSO: data interpretation and data review/approval, and writing of the manuscript, and final approval of the manuscript. SM: planning study design, data interpretation and data review/approval, and writing of the manuscript, and final approval of the manuscript. JS: data interpretation and data review and writing of the manuscript, and final approval of the manuscript. NE: conducting study and data interpretation and writing of the manuscript, and final approval of the manuscript.

Funding This study was supported by a non-profit organization Epidemiological and Clinical Research Information Network (ECRIN).

\section{Compliance with ethical standards}

Conflict of interest Masashi Sawa received honoraria from BristolMyers Squibb. Hiroshi Kojima received honoraria from Celgene. Satoshi Morita received honoraria from Bristol-Myers Squibb. Junichi Sakamoto received honoraria from Tsumura and Chugai Pharmaceutical, and has received consultancy fees from Takeda. Nobuhiko Emi received honoraria from Kyowa Hakko Kirin and Chugai Pharmaceutical. All other authors report no conflicts of interest relevant to this article.

Ethical approval The present study was approved by the Institutional Review Board of the Fujita Health University School of Medicine and conformed to the provisions of the 1964 Declaration of Helsinki and its later amendments or comparable ethical standards. All patients provided written, informed consent before participating in the study.

Open Access This article is distributed under the terms of the Creative Commons Attribution 4.0 International License (http://creativeco mmons.org/licenses/by/4.0/), which permits unrestricted use, distribution, and reproduction in any medium, provided you give appropriate credit to the original author(s) and the source, provide a link to the Creative Commons license, and indicate if changes were made. 


\section{References}

1. Apperley JF (2015) Chronic myeloid leukaemia. Lancet 385:1447-1459

2. Hochhaus A, Baccarani M, Deininger M et al (2008) Dasatinib induces durable cytogenetic responses in patients with chronic myelogenous leukemia in chronic phase with resistance or intolerance to imatinib. Leukemia 22:1200-1206

3. Radich JP, Kopecky KJ, Appelbaum FR et al (2012) A randomized trial of dasatinib $100 \mathrm{mg}$ versus imatinib $400 \mathrm{mg}$ in newly diagnosed chronic phase chronic myeloid leukemia. Blood 120:3898-3905

4. Kantarjian HM, Shah NP, Cortes JE et al (2012) Dasatinib or imatinib in newly diagnosed chronic-phase chronic myeloid leukemia: 2-year follow-up from a randomized phase 3 trial (DASISION). Blood 119:1123-1129

5. Jabbour E, Kantarjian HM, Saglio G et al (2014) Early response with dasatinib or imatinib in chronic myeloid leukemia: 3-year follow-up from a randomized phase 3 trial (DASISION). Blood 123:494-500

6. Cortes JE, Saglio G, Kantarjian HM et al (2016) Final 5-year study results of DASISION: the dasatinib versus imatinib study in treatment-naive chronic myeloid leukemia patients trial. J Clin Oncol 34:2333-2340

7. Marin D, Bazeos A, Mahon FX et al (2010) Adherence is the critical factor for achieving molecular responses in patients with chronic myeloid leukemia who achieve complete cytogenetic responses on imatinib. J Clin Oncol 28:2381-2388

8. Larson RA, Druker BJ, Guilhot F et al (2008) Imatinib pharmacokinetics and its correlation with response and safety in chronicphase chronic myeloid leukemia: a subanalysis of the IRIS study. Blood 111:4022-4028

9. Hughes T, Deininger M, Hochhaus A et al (2006) Monitoring CML patients responding to treatment with tyrosine kinase inhibitors: review and recommendations for harmonizing current methodology for detecting BCR-ABL transcripts and kinase domain mutations and for expressing results. Blood 108:28-37

10. De Francia S, D'Avolio A, De Martino F et al (2009) New HPLCMS method for the simultaneous quantification of the antileukemia drugs imatinib, dasatinib, and nilotinib in human plasma. J Chromatogr B Anal Technol Biomed Life Sci 877:1721-1726
11. Noens L, Hensen M, Kucmin-Bemelmans I et al (2014) Measurement of adherence to BCR-ABL inhibitor therapy in chronic myeloid leukemia: current situation and future challenges. Haematologica 99:437-447

12. Steegmann JL, Baccarani M, Breccia M et al (2016) European Leukemia Net recommendations for the management and avoidance of adverse events of treatment in chronic myeloid leukemia. Leukemia 30:1648-1671

13. Jabbour E, Deininger M, Hochhaus A (2011) Management of adverse events associated with tyrosine kinase inhibitors in the treatment of chronic myeloid leukemia. Leukemia 25:201-210

14. Jabbour EJ, Kantarjian H, Eliasson L et al (2012) Patient adherence to tyrosine kinase inhibitor therapy in chronic myeloid leukemia. Am J Hematol 87:687-691

15. Latagliata $R$, Breccia $M$, Fava $C$ et al (2013) Incidence, risk factors and management of pleural effusions during dasatinib treatment in unselected elderly patients with chronic myelogenous leukemia. Hematol Oncol 31:103-109

16. Eskazan AE, Eyice D, Kurt EA et al (2014) Chronic myeloid leukemia patients who develop grade I/II pleural effusion under second-line dasatinib have better responses and outcomes than patients without pleural effusion. Leuk Res 38:781-787

17. Miura M (2015) Therapeutic drug monitoring of imatinib, nilotinib, and dasatinib for patients with chronic myeloid leukemia. Biol Pharm Bull 38:645-654

18. Yu H, Steeghs N, Nijenhuis CM et al (2014) Practical guidelines for therapeutic drug monitoring of anticancer tyrosine kinase inhibitors: focus on the pharmacokinetic targets. Clin Pharmacokinet 53:305-325

19. Wang X, Roy A, Hochhaus A et al (2013) Differential effects of dosing regimen on the safety and efficacy of dasatinib: retrospective exposure-response analysis of a Phase III study. Clin Pharmacol 5:85-97

20. Rousselot P, Mollica L, Guerci-Bresler A et al (2014) Dasatinib daily dose optimization based on residual drug levels resulted in reduced risk of pleural effusions and high molecular response rates: final results of the randomized OPTIM dasatinib Trial. Abstract ABSSUB-5297, Congress of the European Hematology Association (EHA)

\section{Affiliations}

\section{Shuichi Mizuta ${ }^{1,11}$ - Masashi Sawa ${ }^{2} \cdot$ Hisashi Tsurumi ${ }^{3} \cdot$ Kana Matsumoto $^{4} \cdot$ Kotaro Miyao $^{2} \cdot$ Takeshi Hara $^{3} \cdot$ Takeshi Takahashi $^{5} \cdot$ Reona Sakemura $^{2} \cdot$ Hiroshi Kojima $^{6} \cdot$ Akio Kohno $^{7} \cdot$ Mari S. Oba $^{8} \cdot$ Satoshi Morita ${ }^{9}$. Junichi Sakamoto ${ }^{10} \cdot$ Nobuhiko Emi $^{1}$}

\author{
Masashi Sawa \\ msawa@wine.plala.or.jp \\ Hisashi Tsurumi \\ htsuru@gifu-u.ac.jp \\ Kana Matsumoto \\ kmatsumo@dwc.doshisha.ac.jp \\ Kotaro Miyao \\ koutarou380@yahoo.co.jp \\ Takeshi Hara \\ haratake@muh.biglobe.ne.jp \\ Takeshi Takahashi \\ ttakahashi@gmhosp.gifu.gifu.jp
}

\author{
Reona Sakemura \\ r.sakemura@med.nagoya-u.ac.jp \\ Hiroshi Kojima \\ kojima@daidohp.or.jp \\ Akio Kohno \\ akohno@wd5.so-net.ne.jp \\ Mari S. Oba \\ mari.oba@med.toho-u.ac.jp \\ Satoshi Morita \\ smorita@kuhp.kyoto-u.ac.jp \\ Junichi Sakamoto \\ sakamjun@med.nagoya-u.ac.jp
}


Nobuhiko Emi

nemi@fujita-hu.ac.jp

1 Department of Hematology, Fujita Health University School of Medicine, Toyoake, Japan

2 Department of Hematology and Oncology, Anjo Kosei Hospital, Anjo, Japan

3 Department of Hematology, Graduate School of Medicine, Gifu University, Gifu, Japan

4 Department of Clinical Pharmaceutics, Doshisha Women's College of Liberal Arts, Kyoto, Japan

5 Department of Hematology, Gifu Municipal Hospital, Gifu, Japan
Department of Hematology and Oncology, JA Aichi Konan Kosei Hospital, Konan, Japan

8 Department of Medical Statistics, Faculty of Medicine, Toho University, Tokyo, Japan

9 Department of Biomedical Statistics and Bioinformatics, Kyoto University Graduate School of Medicine, Kyoto, Japan

10 Tokai Central Hospital, Kagamigahara, Japan

11 Hematology and Immunology, Kanazawa Medical University, 1-1 Daigaku, Uchinada, Kahoku-gun, Ishikawa 920-0293, Japan 